Original Study

\title{
Potentially Inappropriate Prescribing in Belgian Nursing Homes: Prevalence and Associated Factors
}

\author{
Pauline M.S. Anrys MS, PhD ${ }^{\mathrm{a}, *}$, Goedele C. Strauven MS, $\mathrm{PhD}^{\mathrm{b}}$, Veerle Foulon MS, $\mathrm{PhD}^{\mathrm{b}}$, \\ Jean-Marie Degryse $\mathrm{MD}, \mathrm{PhD}^{\mathrm{c}, \mathrm{d}}$, Séverine Henrard MS, $\mathrm{PhD}^{\mathrm{a}, \mathrm{c}}$, \\ Anne Spinewine MS, $\mathrm{PhD}^{\mathrm{a}, \mathrm{e}}$
}

a Université catholique de Louvain, Louvain Drug Research Institute, Clinical Pharmacy Research Group, Brussels, Belgium

${ }^{\mathrm{b}}$ KU Leuven, Department of Pharmaceutical and Pharmacological Sciences, Leuven, Belgium

${ }^{\mathrm{c}}$ Université catholique de Louvain, Institute of Health and Society, Brussels, Belgium

${ }^{\mathrm{d}}$ KU Leuven, Department of Public Health and Primary Care, Leuven, Belgium

e Université catholique de Louvain, CHU UCL Namur, Pharmacy department, Yvoir, Belgium

\section{Keywords:}

Inappropriate prescribing

nursing homes

STOPP/START

Beers criteria

\begin{abstract}
A B S T R A C T
Background/objectives: Our aim was to describe the prevalence of potentially inappropriate medications (PIMs) and potential prescribing omissions (PPOs) in Belgian nursing homes and to identify characteristics of residents, general practitioners (GPs), and nursing homes ( $\mathrm{NHs}$ ) that are associated with the number of PIMs and PPOs.

Design: A cross-sectional study.

Setting: and Participants: Nursing home residents (NHRs), aged $\geq 65$ years, not in palliative care were included in 54 Belgian NHs participating in the COME-ON study.

Measures: Instances of PIMs were detected using a combination of the STOPP v2 and AGS 2015 Beers criteria. Instances of PPOs were detected using START v2. To assess factors associated with the number of PIMs and PPOs, a multivariate binomial negative regression analysis was performed.

Results: A total of 1410 residents, with a median age of 87 years, was included. The median number of medications taken was 9. PIMs were detected in $88.3 \%$ of NHRs and PPOs in $85.0 \%$. Use of benzodiazepines (46.7\%) and omission of vitamin D (51.5\%) were the most common PIM and PPO, respectively. The factor most strongly associated with increased PIMs was the use of 5 to 9 drugs or $\geq 10$ drugs [relative risk (RR) (95\% confidence interval [CI]: $2.27(1.89,2.76)$ and $4.04(3.37,4.89)$, respectively]. The resident's age was associated with both decreased PIMs and increased PPOs. PIMs and PPOs were also associated with some NH characteristics, but not with GP characteristics.

Conclusion: Implications: The high prevalence of PIMs and PPOs remains a major challenge for the $\mathrm{NH}$ setting. Future interventions should target in priority residents taking at least 10 medications and/or those taking psychotropic drugs. Future studies should explore factors related to organizational and prescribing culture. Moreover, special attention must be paid to the criteria used to measure inappropriate prescribing, including criteria relative to underuse.
\end{abstract}

(c) 2018 AMDA - The Society for Post-Acute and Long-Term Care Medicine.
This study is funded by the National Institute for Health and Disability Insurance (NIHDI) (Belgium). The funding body had a limited role in the design, but had no role in data collection, implementation, analysis, or the decision to publish the results. PA and GS were paid by the NIHDI.

The authors declare no conflicts of interest.

* Address correspondence to Pauline M.S. Anrys, MS, PhD, Clinical Pharmacy Research Group, Louvain Drug Research Institute, Université catholique de Louvain, Av E Mounier, 72 B1.72.02, Brussels 1200, Belgium.

E-mail address: pauline.anrys@uclouvain.be (P.M.S. Anrys).
Inappropriate prescribing, defined as suboptimal medication use, encompasses 3 categories: overprescribing, that is, the prescription of a drug without a valid indication; misprescribing, that is, incorrectly prescribing a drug for a valid indication; and underprescribing, that is, the failure to prescribe indicated drugs. ${ }^{1}$ Explicit and implicit tools to assess the appropriateness of prescribing are available. The most widely used explicit tools are the Beers ${ }^{2}$ and STOPP-START criteria. $^{3}$ Both were updated in 2015. These tools make it possible to identify potentially inappropriate prescribing (PIP), in the form of potentially inappropriate medications (PIMs, addressed by Beers and STOPP) and/ 
or potential prescribing omissions (PPOs, addressed by START). A high prevalence of PIP has been found across settings $\mathrm{s}^{4,5}$ and in particular in nursing homes (NHs). ${ }^{6,7}$ Moreover, the use of PIMs in the NH setting has been associated with poor outcomes. ${ }^{8}$

Two recent systematic reviews analyzed the PIP use in the $\mathrm{NH}$ setting. The overall PIP prevalence was $43.2 \%,{ }^{6}$ but prevalence varied considerably across studies. ${ }^{7}$ A higher prevalence was observed in Europe than in North America. ${ }^{6}$ However, the studies used older versions of the Beers and STOPP-START criteria, and data on potential underuse were very limited. In Belgium, a study conducted in 2005 reported a prevalence of PIM of $27.0 \%$ according to Beers and a prevalence of PPO of $58.0 \%$ according to ACOVE. ${ }^{9}$ However, the researchers only had access to a limited list of comorbidities and did not apply the "unless" rules of the criteria. Furthermore, the 2003 version of the Beers criteria has been criticized for its restricted applicability in Europe. $^{10}$

In the quest for the optimization of medication use in the $\mathrm{NH}$ setting, identifying factors associated with inappropriate prescribing might be of great value, as it could show the factors to target and the NHRs who might benefit most. The most common factors previously found to be associated with PIMs in NHs are polypharmacy, ${ }^{6,11}$ geographic region, ${ }^{11}$ younger age, ${ }^{11}$ and diagnoses of depression or diabetes. ${ }^{11}$ A very limited number of studies has explored the association between PIMs and NH or general practitioner (GP) characteristics. Moreover, factors associated with PPOs have not been extensively studied. To our knowledge, no investigation has been carried out in the NH setting.

In the present study, we aimed (1) to describe the prevalence of PIMs and PPOs in a sample of NHRs in Belgium and (2) to identify which factors - at the resident, GP, and NH levels-are associated with the number of PIMs or PPOs.

\section{Methods}

\section{Study Setting}

The present work is a cross-sectional analysis of the baseline data of the COME-ON study. This multicenter cluster-controlled trial was conducted in 54 NHs in Belgium (37 in Flanders and 17 in Wallonia) with the aim of assessing the impact of a complex intervention on the appropriateness of prescribing. The COME-ON study protocol has been described elsewhere. ${ }^{12}$ In each $\mathrm{NH}$, the aim was to recruit 35 NHRs aged 65 years and older, under the care of a participating GP.

The health care professionals (ie, GP, nurse, and pharmacist) caring for each resident prospectively recorded data (ie, clinical data, medical conditions, laboratory values, and the medication schedule) in a dedicated secured web application.

Each Belgian NH has to appoint a coordinating physician (CP) who is responsible for training, coordination of quality initiatives, etc. Residents can choose their GP; consequently, the number of visiting GPs is unrestricted. Furthermore, the GP has total freedom in the choice of therapeutic strategies. The delivery of medication is performed by a hospital or community pharmacy. Drugs are delivered either in their original boxes or using multidose drug dispensing (ie, 1 unit for each dose occasion is packed in individual bags). Currently, there is no legal obligation to conduct medication reviews in NHs, and the role of the pharmacist is mainly focused on the delivery of medications.

\section{Study Sample}

From the 1804 NHRs included in the COME-ON study, data required for the identification of PIP at baseline (ie, clinical data, comorbidities, and medication schedule) were available for 1507 NHRs. Ninety-seven NHRs in palliative care at the time of data collection were excluded. Therefore, a total of 1410 NHRs were included in this analysis (Figure 1). Some clinical data or comorbidities could be registered as "unknown/I don't know" by the nurse or the GP. In the analysis, we considered an "unknown/I don't know" variable as "not present."

\section{Identification of PIMs and PPOs}

PIMs and PPOs were automatically detected using an algorithm specially developed for the study. ${ }^{13}$ The STOPP-START version $2^{3}$ and the AGS 2015 Beers criteria ${ }^{2}$ were applied. As previously described, ${ }^{13}$

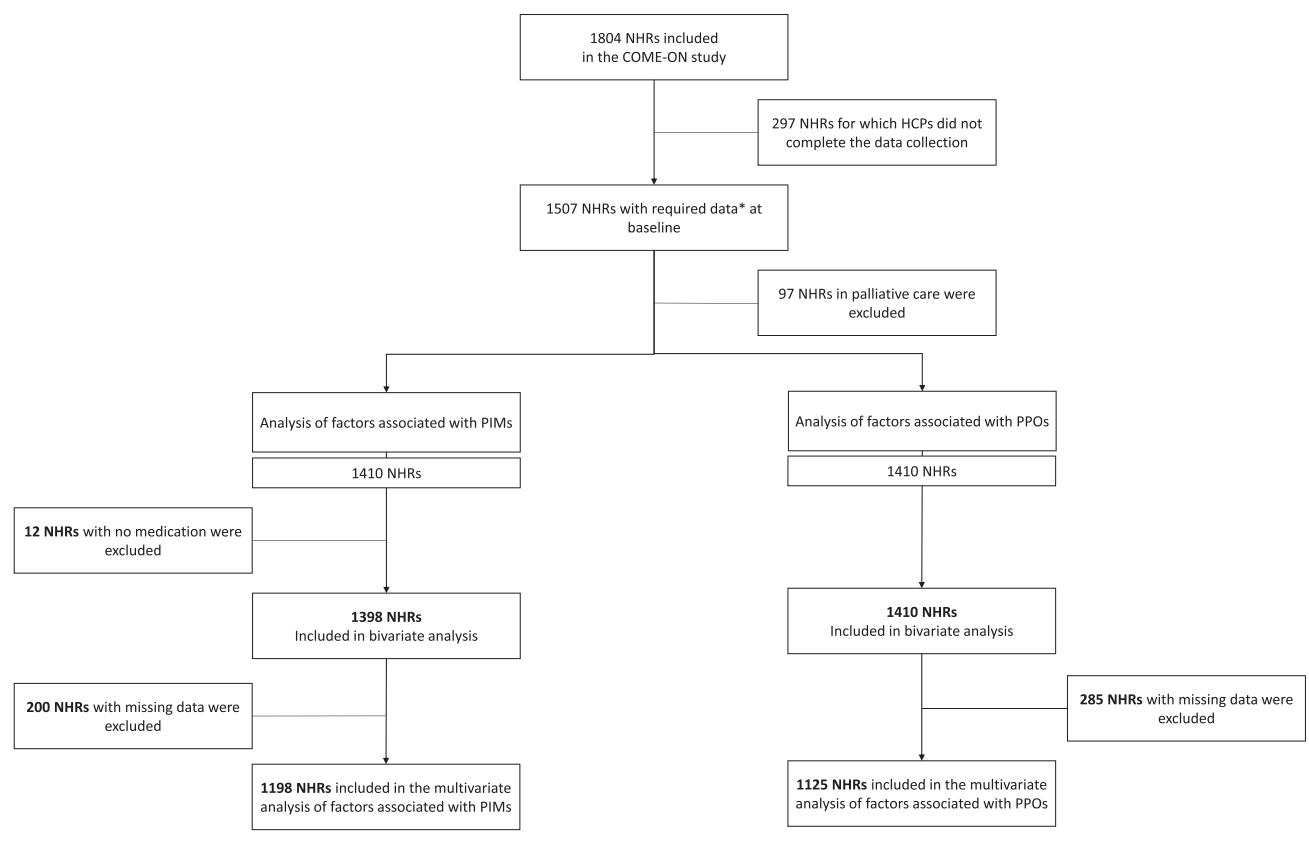


some adaptations were made while designing the algorithm, including the exclusion of some criteria not applicable in an algorithm or criteria that include medications not available in Belgium. Finally, $95 \%(n=76 / 80)$ of STOPP, $91 \%(n=31 / 34)$ of START, and $84 \%(n=71 /$ 85) of Beers criteria were included (Appendix Table 1). Instances of PIM were detected using a combination of STOPP and Beers criteria for NHRs taking at least 1 medication. The START criteria were applied to all NHRs. PIM instance were considered at the level of the medication. For example, if an NHR taking 1 NSAID met conditions for STOPP H1 and STOPP H2, then the NSAID was considered as 1 PIM.

\section{Selection of Covariates}

The selection of factors for which the association with PIMs and PPOs was going to be tested was made based on literature data as well as on relevance as judged by the research team.

\section{NHR Characteristics}

Ten NHR characteristics were tested for association with PIP: age, gender, number of medications, time since institutionalization, Katz score, Cumulative Illness Rating Scale for Geriatrics, neuropsychiatric status, history of falls and hospitalization in the previous 3 months, and delirium. The categories for the number of years since institutionalization, Katz score, and Cumulative Illness Rating Scale for Geriatrics score were defined using a regression tree. ${ }^{14}$ Neuropsychiatric status was divided into 3 categories: (1) no neuropsychiatric disorder, (2) dementia or cognitive impairment (as reported by GP), and (3) other neuropsychiatric disorder, such as anxiety, depression, and psychosis, without dementia/cognitive impairment. ${ }^{15}$ The number of medications included all medications taken (ie, prescribed and nonprescribed drugs, including chronic, acute, as-needed, and compounded medications). Three categories were used: $<5,5$ to 9 , and $\geq 10$ medications. The scale used to compute the Katz score is a modified version of the original scoring system used in Belgium and has 4 categories, ranging from 1 for independence to 4 for dependence.

\section{GP Characteristics}

Four GP characteristics thought to have a possible influence on prescribing patterns were included in the analysis: the GP's role (GP or coordinating physician), practice (single-handed practice or group practice), collaboration with GP trainee, and number of years since graduation (categorized using a regression tree ${ }^{14}$ ).

\section{NH Characteristics}

Seven characteristics of NHs were evaluated: region, ownership status, number of beds (categories were based on data previously published in Belgium ${ }^{16}$ ), nurse (full-time equivalent) to residents ratio, previous experience of some form of case conference, the use of multidose drug dispensing, and the proportion of NHRs for which the coordinating physician of the $\mathrm{NH}$ is the treating $\mathrm{GP}$ (categorized using a regression tree ${ }^{14}$ ).

\section{Statistical Analysis}

In the descriptive analyses, continuous data were expressed as median (P25-P75) and categorical data were summarized using proportions. The prevalence of PIMs and PPOs were described at the level of the criteria and of the NHR. Given the high proportion of residents with $\geq 1$ PIM (88.3\%) or PPO (85.0\%), PIMs and PPOs were considered as discrete count outcome variables instead of a binary variable, that is, presence of $\geq 1$ PIM or PPO. Outcome variables were expressed as rate of PIMs or PPOs, that is, the average number of PIMs or PPOs per resident. To take into account the overdispersion of these count outcome variables, a negative binomial regression was performed. First, a bivariate analysis was conducted for the association between the outcomes and characteristics of NHRs, GPs, and NHs as covariates.

Second, variables for which an association with the outcomes was found in the bivariate model with a $P \leq .15$, were included as candidates for the final multivariable model, after testing for multicollinearity. Where there was multicollinearity [variance inflation factor $(\mathrm{VIF})>6$ ], the variable to be kept in the model was chosen based on clinical arguments. A stepwise approach was used to select the final multivariable model based on the Akaike information criterion (AIC). Finally, the goodness of fit of each final model was assessed using Pearson chi-squared goodness-of-fit test.

To take into account the hierarchical structure of the data (level 1: NHRs, level 2: GPs, level 3: NHs), mixed-effect models were tested. As no significant variability between NHs and GPs on either outcomes was present, negative binomial regression models, not taking into account the hierarchical structure of the data, were used.

A $P$ value of $<.05$ was considered statistically significant. All statistical analyses were performed using R software version 3.3.1 (Free Software Foundation, Inc, Boston, MA).

\section{Ethical Considerations}

This study was approved by the Ethics Committee of UZ Leuven (s57145, ML11035) and by the Belgian Privacy Commission (SCSZ/14/ 084/174). All the NHRs or residents' representatives provided written informed consent.

\section{Study Registration}

This study has been registered at http://www.isrctn.com/ (trial registration number: ISRCTN66138978).

\section{Results}

\section{Characteristics of NHRs}

The median age was 87 years; $72.0 \%$ were women. The median Cumulative Illness Rating Scale for Geriatrics score was 9. The number of medications per NHR ranged from 0 to 25, with a median of 9 . Characteristics of NHRs are presented in Table 1 and characteristics of GPs and NHs are displayed in Appendix Table 2.

\section{Prevalence of PIMs and PPOs}

The presence of at least 1 PIM, as determined by the STOPP v2 and/ or the AGS 2015 Beers criteria, was detected in $88.3 \%(n=1245)$ of NHRs. The median number of PIMs was $2 .^{1-4}$ Psychotropic agents, anticholinergic drugs, and pump-proton inhibitors were the most frequent PIMs. The 10 most prevalent PIMs are listed in Appendix Table 3.

The presence of at least 1 PPO according to START v2 was detected in $85.0 \%(\mathrm{n}=1199)$ of NHRs. The median number of PPOs was $2 .^{1-3}$ The main conditions for which potential underuse was detected were osteoporosis and cardiovascular prevention. The 10 most prevalent PPOs are listed in Appendix Table 4.

\section{Factors Associated With PIMs}

In the final multivariable model, $4 \mathrm{NHR}$ characteristics and $2 \mathrm{NH}$ characteristics, but no GP characteristics, were associated with number of PIMs (Table 2). 
Table 1

Characteristics of Nursing Home Residents and Overall PIM/PPO Prevalence

\begin{tabular}{|c|c|}
\hline & $\begin{array}{l}\text { Total }(\mathrm{N}=1410) \\
\text { Median }\left[\mathrm{P}_{25}-\mathrm{P}_{75}\right] \\
\text { or } \mathrm{n}(\%)\end{array}$ \\
\hline Age, in years* & 87 [82-91] \\
\hline $65-74$ & $100(7.1)$ \\
\hline $75-84$ & $417(29.8)$ \\
\hline$\geq 85$ & $884(63.1)$ \\
\hline \multicolumn{2}{|l|}{ Gender } \\
\hline Women & $1012(71.8)$ \\
\hline Men & $398(28.2)$ \\
\hline Duration since institutionalization, years ${ }^{\dagger}$ & $2[1-5]$ \\
\hline$\leq 2$ & $720(51.6)$ \\
\hline $3-6$ & $454(32.5)$ \\
\hline $7-12$ & $164(11.7)$ \\
\hline$\geq 13$ & $59(4.2)$ \\
\hline Katz score & $17[13-20]$ \\
\hline$\leq 10$ & $190(15.4)$ \\
\hline $11-15$ & $321(26.1)$ \\
\hline 16-19 & $329(26.7)$ \\
\hline$\geq 20$ & $392(31.8)$ \\
\hline CIRS-G score & $9[6-13]$ \\
\hline$\leq 6$ & $403(28.6)$ \\
\hline $7-10$ & $425(30.1)$ \\
\hline $11-16$ & $421(29.9)$ \\
\hline$\geq 17$ & $161(11.4)$ \\
\hline History of falls in the previous $3 \mathrm{mo}$ & $325(23.0)$ \\
\hline Hospitalization in the previous 3 mo & $140(9.9)$ \\
\hline \multicolumn{2}{|l|}{ Neuropsychiatric status } \\
\hline No neuropsychiatric disorders & $356(25.2)$ \\
\hline $\begin{array}{l}\text { Any dementia, including Alzheimer's disease, Lewy } \\
\text { body disease, other type of dementia, cognitive } \\
\text { impairment }\end{array}$ & $781(55.4)$ \\
\hline $\begin{array}{l}\text { Other neuropsychiatric disorders only, such as } \\
\text { anxiety disorder, depression, psychosis }\end{array}$ & $273(19.4)$ \\
\hline Number of medications & $9[6-12]$ \\
\hline$<5$ & $172(12.2)$ \\
\hline 5-9 & $601(42.6)$ \\
\hline$\geq 10$ & $637(45.2)$ \\
\hline \multicolumn{2}{|l|}{$\begin{array}{l}\text { The } 10 \text { most prescribed medication classes } \\
\text { (ATC level 2) }\end{array}$} \\
\hline Psycholeptics (N05) & $923(65.5)$ \\
\hline Antithrombotic agents (B01) & $822(58.3)$ \\
\hline Analgesics (N02) & $768(54.5)$ \\
\hline Drugs for constipation (A06) & $746(52.9)$ \\
\hline Psychoanaleptics (N06) & $681(48.3)$ \\
\hline Drugs for acid-related disorders (A02) & $645(45.7)$ \\
\hline Vitamins (A11) & $583(41.3)$ \\
\hline Beta-blocking agents (C07) & $562(39.9)$ \\
\hline Diuretics (C03) & $538(38.2)$ \\
\hline $\begin{array}{l}\text { Agents acting on the renin-angiotensin system } \\
\text { (C09) }\end{array}$ & $415(29.4)$ \\
\hline Mineral supplements (A12) & $354(25.1)$ \\
\hline \multicolumn{2}{|l|}{ Prevalence of PIMs } \\
\hline NHRs with at least 1 PIM & $1245(88.3)$ \\
\hline Number of PIMs & $2[1-4]$ \\
\hline \multicolumn{2}{|l|}{ Prevalence of PPOs } \\
\hline NHRs with at least 1 PPO & $1199(85.0)$ \\
\hline Number of PPOs & $2[1-3]$ \\
\hline
\end{tabular}

ATC, Anatomical Therapeutic Chemical Classification; CIRS-G, Cumulative Illness Rating Scale; PIMs, potentially inappropriate medications; PPOs, potential prescribing omissions.

${ }^{*}$ Missing data: $\mathrm{n}=9(0.6 \%)$.

Missing data: $\mathrm{n}=13(0.9 \%)$.

Missing data: $\mathrm{n}=178(12.6 \%)$.

The strongest predictor of a higher rate of PIMs was the number of medications. Residents with a history of falls and those suffering from dementia/cognitive impairment or from other neuropsychiatric disorders (ie, anxiety, depression and psychosis) also had a higher rate of PIMs. Furthermore, residing in Wallonia and being in a private forprofit $\mathrm{NH}$ were associated with higher rates of PIMs. Conversely, the oldest NHRs ( $\geq 85$ years) had a lower rate of PIMs than residents aged 65 to 74 years.

\section{Factors Associated With PPOs}

In the final multivariable model, $4 \mathrm{NHR}$ characteristics and $1 \mathrm{NH}$ characteristic, but no GP characteristics, were associated with the number of PPOs (Table 3).

Older age, increasing comorbidity burden, and higher dependency (ie, Katz score $\geq 20$ ) were associated with higher rates of PPOs. Residents who had been institutionalized for 3 to 6 years and NHRs living in NHs in which the CP takes over the care of more than half of NHRs, had lower rates of PPOs.

\section{Discussion}

Our study shows that the prevalence of PIP in Belgian NHRs is high, as about $90 \%$ and $85 \%$ of NHRs had at least 1 PIM and PPO, respectively. Several resident and $\mathrm{NH}$ characteristics were associated with PIMs and PPOs, while no association was found with GP characteristics. Age is the only factor that was associated with both PIMs and PPOs, but inversely.

This is the first study using the latest update of the STOPP-START and Beers criteria to evaluate the association between 3 types of factors and PIMs and PPOs. We had clinical data and data on comorbidities; this allowed us to apply the great majority of explicit criteria.

PIM and PPO prevalence was similar to or even higher than the highest prevalence reported in the literature. ${ }^{6,7}$ Irrespective of the prevalence, the main drug classes involved were similar to those reported in the literature. ${ }^{17,18}$ There can be various reasons for the higher rate of PIP found in the present study. The tool(s) used to measure PIP and the residents' information available may influence the results. We used 2 different tools, and had comprehensive information (including on diagnose) to identify PIPs, which may lead to a higher prevalence. Variations in prevalence rates of PIP between studies also may be related to differences in the population that is studied. ${ }^{19}$ Importantly, differences may also arise because some countries have implemented national strategies to tackle inappropriate use, including antipsychotic drugs use. ${ }^{4}$

With regard to NHR characteristics associated with PIMs, not surprisingly, a greater number of medications has been consistently identified as the strongest determinant of PIM use in the $\mathrm{NH}$ setting ${ }^{15,20-27}$ and across settings ${ }^{11}$; this is consistent with our results. Similarly, younger age has been associated with a higher rate of PIMs. ${ }^{11,22,23,28}$ The oldest residents may have fewer comorbidities, reflecting a "survival of the fittest." ${ }^{22,23}$ Furthermore, GPs may be more cautious in prescribing because they see very old residents as frailer. ${ }^{15,22}$ However, many studies in NHs have not demonstrated this association. 15,24,26,27,29-31

The association between PIMs and a previous fall(s) may be explained by the fact that the most common PIMs were benzodiazepines, Z-drugs, antipsychotics, and anticholinergic drugs: these drugs have been associated with an increased risk of falls in NHRs. ${ }^{32,33}$ To our knowledge, only 2 studies have investigated the association between falls or risk of falls and PIM use, and their conclusions differed. $^{30,31}$ Similarly, medications that act on the central nervous system contribute to many PIMs and this may explain the association between PIMs and neuropsychiatric disorders. ${ }^{15}$ As for the association between dementia and PIMs, most previous studies did not find an association, ${ }^{15,20,27,31}$ and one study found an inverse relationship. ${ }^{28}$

Studies investigating the impact of GPs' characteristics on PIP prescribing are lacking. ${ }^{11}$ In a study conducted in primary care, Amos et al reported that patients under the care of older GPs, GPs working solo, and GPs caring for fewer older patients were more likely to receive at least 1 PIM. ${ }^{34}$ In our study, no GP characteristics were found to be significantly associated with PIMs or PPOs in the multivariate analysis. We could assume that GP characteristics may have less influence for institutionalized patients. Alternatively, other factors 
Table 2

Factors Associated With Potentially Inappropriate Medications (PIMs) in Bivariate and Multivariable Analysis

\begin{tabular}{|c|c|c|c|c|c|c|}
\hline \multirow[t]{2}{*}{ Variable } & \multicolumn{3}{|c|}{ Bivariate Analysis, $\mathrm{n}=1398$} & \multicolumn{3}{|c|}{ Multivariable Analysis, $\mathrm{n}=1198$} \\
\hline & $\mathrm{n}$ & $\mathrm{RR}(95 \% \mathrm{CI})$ & $P$ Value & $\mathrm{n}$ & $\mathrm{RR}(95 \% \mathrm{CI})$ & $P$ Value \\
\hline \multicolumn{7}{|l|}{ Characteristics of nursing home residents } \\
\hline \multicolumn{7}{|l|}{ Age, $\mathrm{y}^{*}$} \\
\hline $65-74$ & 98 & 1.00 & & 83 & 1.00 & \\
\hline $75-84$ & 413 & $0.85(0.74,0.98)$ & .028 & 362 & $0.88(0.76,1.01)$ & .060 \\
\hline$\geq 85$ & 878 & $0.74(0.65,0.85)$ & $<.001$ & 753 & $0.82(0.72,0.93)$ & .002 \\
\hline \multicolumn{7}{|l|}{ History of falls in the previous $3 \mathrm{mo}$} \\
\hline No & 1074 & 1.00 & & 917 & 1.00 & \\
\hline Yes & 324 & $1.24(1.14,1.35)$ & $<.001$ & 281 & $1.18(1.09,1.28)$ & $<.001$ \\
\hline \multicolumn{7}{|l|}{ Neuropsychiatric status } \\
\hline No neuropsychiatric disorders & 352 & 1.00 & & 295 & 1.00 & \\
\hline Any dementia or cognitive impairment & 774 & $1.18(1.08,1.29)$ & $<.001$ & 677 & $1.18(1.08,1.30)$ & $<.001$ \\
\hline Only other neuropsychiatric disorders: anxiety, depression, psychosis & 272 & $1.54(1.38,1.71)$ & $<.001$ & 226 & $1.27(1.14,1.41)$ & $<.001$ \\
\hline \multicolumn{7}{|l|}{ Number of medications } \\
\hline$<5$ & 160 & 1.00 & & 144 & 1.00 & \\
\hline $5-9$ & 601 & $2.29(1.93,2.75)$ & $<.001$ & 509 & $2.27(1.89,2.76)$ & $<.001$ \\
\hline$\geq 10$ & 637 & $4.27(3.60,5.11)$ & $<.001$ & 545 & $4.04(3.37,4.89)$ & $<.001$ \\
\hline \multicolumn{7}{|l|}{ Characteristics of nursing homes } \\
\hline \multicolumn{7}{|l|}{ Region } \\
\hline Flanders & 976 & 1.00 & & 878 & 1.00 & \\
\hline Wallonia & 422 & $1.29(1.19,1.39)$ & $<.001$ & 320 & $1.19(1.09,1.21)$ & $<.001$ \\
\hline \multicolumn{7}{|l|}{ Ownership status } \\
\hline Private nonprofit & 909 & 1.00 & & 807 & 1.00 & \\
\hline Private for profit & 151 & $1.20(1.06,1.34)$ & .002 & 95 & $1.19(1.04,1.36)$ & .010 \\
\hline Public & 338 & $1.15(1.05,1.25)$ & .002 & 296 & $1.07(0.98,1.17)$ & .107 \\
\hline
\end{tabular}

CI, confidence interval; CIRS-G: Cumulative Illness Rating Scale for Geriatrics; RR, rate ratio.

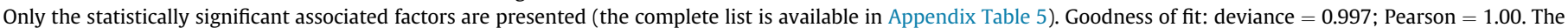

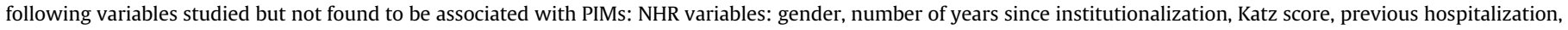

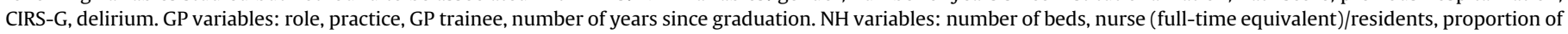
residents for which the $\mathrm{GP}$ is the $\mathrm{CP}$, previous experience of case conferences, multidose drug dispensing.

*Missing data: $\mathrm{n}=9$ (0.6\%).

Table 3

Factors Associated With Potential Prescribing Omissions (PPOs) in Bivariate and Multivariable Analysis

\begin{tabular}{|c|c|c|c|c|c|c|}
\hline \multirow[t]{2}{*}{ Variable } & \multicolumn{3}{|c|}{ Bivariate Analysis, $\mathrm{n}=1410$} & \multicolumn{3}{|c|}{ Multivariable Analysis, $\mathrm{n}=1125$} \\
\hline & $\mathrm{n}$ & $\mathrm{RR}(95 \% \mathrm{CI})$ & $P$ Value & $\mathrm{n}$ & $\mathrm{RR}(95 \% \mathrm{CI})$ & $P$ Value \\
\hline \multicolumn{7}{|c|}{ Characteristics of nursing home residents } \\
\hline \multicolumn{7}{|c|}{ Age, $\mathrm{y}^{*}$} \\
\hline $65-74$ & 100 & 1.00 & & 73 & 1.00 & \\
\hline $75-84$ & 417 & $1.20(1.01,1.44)$ & .047 & 329 & $1.31(1.07,1.63)$ & .012 \\
\hline$\geq 85$ & 884 & $1.21(1.02,1.44)$ & .029 & 723 & $1.31(1.07,1.61)$ & .009 \\
\hline \multicolumn{7}{|c|}{ Number of years since institutionalization ${ }^{\dagger}$} \\
\hline$\leq 2$ & 720 & 1.00 & & 575 & 1.00 & \\
\hline $3-6$ & 454 & $0.89(0.81,0.98)$ & .015 & 375 & $0.88(0.80,0.97)$ & .013 \\
\hline $7-12$ & 164 & $1.00(0.87,1.13)$ & .956 & 132 & $0.99(0.86,1.13)$ & .853 \\
\hline$\geq 13$ & 59 & $0.95(0.77,1.17)$ & .658 & 43 & $1.16(0.92,1.44)$ & .201 \\
\hline \multicolumn{7}{|l|}{ Katz score } \\
\hline$\leq 10$ & 190 & 1.00 & & 174 & 1.00 & \\
\hline $11-15$ & 321 & $1.07(0.93,1.25)$ & .348 & 292 & $1.04(0.90,1.21)$ & .578 \\
\hline $16-19$ & 329 & $1.15(0.99,1.33)$ & .063 & 302 & $1.06(0.91,1.23)$ & .468 \\
\hline$\geq 20$ & 392 & $1.34(1.16,1.54)$ & $<.001$ & 357 & $1.22(1.06,1.41)$ & .005 \\
\hline \multicolumn{7}{|c|}{ CIRS-G total score } \\
\hline$\leq 6$ & 403 & 1.00 & & 318 & 1.00 & \\
\hline $7-10$ & 425 & $1.28(1.14,1.43)$ & $<.001$ & 335 & $1.22(1.07,1.38)$ & .002 \\
\hline $11-16$ & 421 & $1.62(1.45,1.80)$ & $<.001$ & 348 & $1.51(1.34,1.71)$ & $<.001$ \\
\hline$\geq 17$ & 161 & $1.81(1.58,2.06)$ & $<.001$ & 124 & $1.72(1.47,2.00)$ & $<.001$ \\
\hline \multicolumn{7}{|c|}{ Characteristics of nursing home } \\
\hline \multicolumn{7}{|c|}{ Proportion of residents for which the GP is the $\mathrm{CP}$} \\
\hline$\leq 10.9 \%$ & 598 & 1.00 & & 458 & 1.00 & \\
\hline $11-25.9 \%$ & 373 & $0.91(0.82,1.00)$ & .054 & 289 & $0.90(0.81,1.01)$ & .076 \\
\hline $26-50.9 \%$ & 377 & $0.91(0.82,1.00)$ & .063 & 348 & $0.94(0.85,1.05)$ & .266 \\
\hline$\geq 51 \%$ & 62 & $0.88(0.71,1.08)$ & .221 & 30 & $0.66(0.46,0.93)$ & .022 \\
\hline
\end{tabular}

$\mathrm{CI}$, confidence interval; RR: rate ratio.

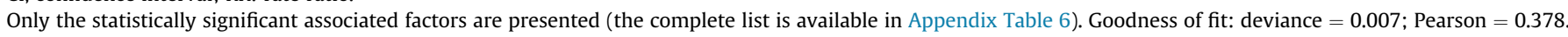

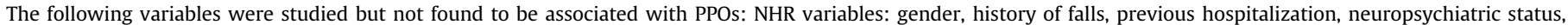

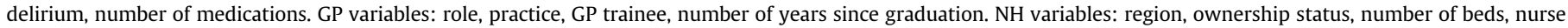
(full-time equivalent)/residents, previous experience of case conferences, multidose drug dispensing.

*Missing data: $\mathrm{n}=9$ (0.6\%).

${ }^{\dagger}$ Missing data: $\mathrm{n}=13(0.9 \%)$.

${ }^{\ddagger}$ Missing data: $\mathrm{n}=178$ (12.6\%). 
related to GPs and to the GP-patient relationship may be more important, but were not available (ie, proportion of NHRs cared for by each GP overall, number of visits ${ }^{35}$ ) or difficult to measure in quantitative research. In previous qualitative research, factors found to influence PIM prescribing included the need to please the patient, a feeling of being forced to prescribe, tension between prescribing experience, and prescribing guidelines. ${ }^{36,37}$

Coordinating physicians receive specific training and have specific roles relative to appropriate medication management in the $\mathrm{NH}$. This factor was associated with a lower number of PIMs in univariate but not multivariate analyses. We may assume that current training and roles may need strengthening, and that other factors more strongly influence the number of PIMs and PPOs.

Very few studies have explored the association between PIMs and $\mathrm{NH}$ characteristics. In contrast to previous studies, we found no association between the ratio nurses/residents with either PIMs or PPOs. This may be due to low variability between NHs in this ratio. Previous experience of medication review was not associated with PIMs in the multivariate model. This needs to be interpreted cautiously, as there is no definition of medication review in Belgium and there was wide variability in content and intensity for those who reported previous experience.

The effect of region deserves specific attention. Even though several individual factors differed between regions (eg, there were fewer NHRs taking at least 10 drugs in Flanders), the effect of region remained significant after taking these differences into account. This may be attributable to the fact that other variables of interest that were not captured in the present analysis play a role, and/or that the combination of several individual factors is important. In a recent systematic review, Morin et al suggested that cultural differences in beliefs, attitudes, and practices and the development of regulation or initiatives to promote the quality of prescribing could explain variation in PIP prevalence between Europe and North America. ${ }^{6}$ We believe that our data concur with this hypothesis, as several initiatives to promote quality of care in the $\mathrm{NH}$ setting have been implemented in Flanders but not in Wallonia. ${ }^{38,39}$ Investigation of organizational and safety culture (including aspects relative to communication, teamwork, training, and family involvement), and of the level of practice of nonpharmacologic management could also provide a better understanding of differences between regions and NHs. ${ }^{40}$

To our knowledge, no previous study has evaluated the factors associated with PPOs according to START criteria in the NH setting. Consistent with previous studies conducted in other settings, comorbidity burden was associated with a higher rate of underprescribing, ${ }^{41-43}$ but no association was found with the number of medications. Interestingly, older age had a protective effect against PIMs but was associated with a higher rate of PPOs. A possible explanation may be GPs' reluctance to add a new drug, especially for long-term prevention, in NHRs who have multimorbidity or are older. Such PIP may therefore prove to be actually appropriate prescribing. The START criteria might not be clinically relevant for NHRs, especially the frailest. The recently developed explicit PIP criteria for NHs in the United States has 24 criteria, of which only 2 are measures of underused medications. $^{44}$

The present study has some limitations. First, the STOPP/START and Beers criteria were developed for older people but are not specifically tailored to the situation of NHRs. Some criteria may have limited value, certainly for people with short life expectancy. ${ }^{18}$ After the present study began, new sets of criteria, specifically tailored to the situation of NHRs and of frail older people, were developed. ${ }^{31,44,45}$ It might be of interest to compare prevalence and associated factors using these new sets, especially for the frailest patients. Second, we identified factors associated with PIP but we did not evaluate actual inappropriate prescribing and related adverse outcomes. Third, a selection bias at different levels cannot be excluded, firstly, as NHs and health care professionals volunteered to participate in the study and may, thus, be more aware than other NHs and health care professionals of the importance of the quality of prescribing. Second, some residents were excluded due either to missing data or to palliative care, this could be a bias because they were older and more dependent.

\section{Conclusion/Relevance}

Despite considerable attention over the last decades, inappropriate prescribing in the $\mathrm{NH}$ setting remains a major challenge. Targeted interventions should focus in priority on NHRs taking at least 10 medications, and possibly those taking psychotropic drugs. Nursing homes and system-related factors, such as regional initiatives to promote quality, may be associated with improved prescribing. However, these factors, together with additional factors relative to the safety culture, need to be further explored. Importantly, future studies in the NH setting should measure PIP using criteria that are relevant to the $\mathrm{NH}$ setting, and the clinical relevance of the START criteria in the $\mathrm{NH}$ setting deserve to be further explored.

\section{Supplementary data}

Supplementary data related to this article can be found at https:// doi.org/10.1016/j.jamda.2018.06.010

\section{References}

1. Spinewine A, Schmader KE, Barber N, et al. Appropriate prescribing in elderly people: How well can it be measured and optimised? Lancet 2007;370: $173-184$.

2. American Geriatrics Society 2015 Beers Criteria Update Expert Panel. American Geriatrics Society 2015 Updated Beers Criteria for potentially inappropriate medication use in older adults. J Am Geriatr Soc 2015;63:2227-2246.

3. O'Mahony D, O'Sullivan D, Byrne S, et al. STOPP/START criteria for potentially inappropriate prescribing in older people: Version 2. Age Ageing 2015;44: $213-218$.

4. Tommelein E, Mehuys E, Petrovic M, et al. Potentially inappropriate prescribing in community-dwelling older people across Europe: A systematic literature review. Eur J Clin Pharmacol 2015;71:1415-1427.

5. Opondo D, Eslami S, Visscher S, et al. Inappropriateness of medication prescriptions to elderly patients in the primary care setting: A systematic review. PLOS One 2012;7:e43617.

6. Morin L, Laroche ML, Texier G, et al. Prevalence of potentially inappropriate medication use in older adults living in nursing homes: A systematic review. J Am Med Dir Assoc 2016;17:862.e1-862.e9.

7. Storms H, Marquet K, Aertgeerts B, et al. Prevalence of inappropriate medication use in residential long-term care facilities for the elderly: A systematic review. Eur J Gen Pract 2017;23:69-77.

8. Morley JE. Inappropriate drug prescribing and polypharmacy are major causes of poor outcomes in long-term care. J Am Med Dir Assoc 2014;15:780-782.

9. Elseviers MM, Vander Stichele RR, Van Bortel L. Quality of prescribing in Belgian nursing homes: An electronic assessment of the medication chart. Int J Qual Health Care 2014;26:93-99.

10. Dalleur O, Boland B, Spinewine A. 2012 updated beers criteria: Greater applicability to Europe? J Am Geriatr Soc 2012;60:2188-2189.

11. Nothelle SK, Sharma R, Oakes AH, et al. Determinants of potentially inappropriate medication use in long-term and acute care settings: A systematic review. J Am Med Dir Assoc 2017;18:806.e1-806.e17.

12. Anrys P, Strauven G, Boland B, et al. Collaborative approach to Optimise MEdication use for Older people in Nursing homes (COME-ON): Study protocol of a cluster controlled trial. Implement Sci 2016;11:35.

13. Anrys P, Boland B, Degryse JM, et al. STOPP/START version 2-development of software applications: Easier said than done? Age Ageing 2016;45:589-592.

14. Kuhn L, Page K, Ward J, et al. The process and utility of classification and regression tree methodology in nursing research. J Adv Nurs 2014;70: $1276-1286$.

15. Lau DT, Kasper JD, Potter DE, et al. Potentially inappropriate medication prescriptions among elderly nursing home residents: Their scope and associated resident and facility characteristics. Health Serv Res 2004;39:1257-1276.

16. KCE. L'utilisation des médicaments dans les maisons de repos et les maisons de repos et de soins belges KCE reports vol. 47B. 2006.

17. Montastruc F, Gardette V, Cantet C, et al. Potentially inappropriate medication use among patients with Alzheimer disease in the REAL.FR cohort: Be aware of atropinic and benzodiazepine drugs! Eur J Clin Pharmacol 2013;69: $1589-1597$. 
18. Ryan C, O'Mahony D, Kennedy J, et al. Potentially inappropriate prescribing in older residents in Irish nursing homes. Age Ageing 2013;42:116-120.

19. Fialova D, Topinkova E, Gambassi G, et al. Potentially inappropriate medication use among elderly home care patients in Europe. JAMA 2005;293: $1348-1358$.

20. Gray SL, Hedrick SC, Rhinard EE, et al. Potentially inappropriate medication use in community residential care facilities. Ann Pharmacother 2003:37:988-993.

21. Rigler SK, Jachna CM, Perera S, et al. Patterns of potentially inappropriate medication use across three cohorts of older Medicaid recipients. Ann Pharmacother 2005;39:1175-1181.

22. Rancourt C, Moisan J, Baillargeon L, et al. Potentially inappropriate prescriptions for older patients in long-term care. BMC Geriatr 2004;4:9.

23. Ruths S. Evaluation of prescribing quality in nursing homes based on drugspecific indicators: The Bergen district nursing home (BEDNURS) study. Norsk Epidemiologi 2008;18:173-178.

24. Stafford AC, Alswayan MS, Tenni PC. Inappropriate prescribing in older residents of Australian care homes. J Clin Pharm Ther 2011;36:33-44.

25. Chen LL, Tangiisuran B, Shafie AA, et al. Evaluation of potentially inappropriate medications among older residents of Malaysian nursing homes. Int J Clin Pharm 2012;34:596-603.

26. Vieira de Lima TJ, Garbin CA, Garbin AJ, et al. Potentially inappropriate medications used by the elderly: Prevalence and risk factors in Brazilian care homes. BMC Geriatr 2013;13:52.

27. Lao CK, Ho SC, Chan KK, et al. Potentially inappropriate prescribing and drugdrug interactions among elderly Chinese nursing home residents in Macao. Int J Clin Pharm 2013;35:805-812.

28. Cool C, Cestac P, Laborde C, et al. Potentially inappropriate drug prescribing and associated factors in nursing homes. J Am Med Dir Assoc 2014;15:850.e1-850. e9.

29. Rigler SK, Perera S, Jachna C, et al. Comparison of the association between disease burden and inappropriate medication use across three cohorts of older adults. Am J Geriatr Pharmacother 2004;2:239-247.

30. Ailabouni NJ, Nishtala PS, Tordoff JM. Examining potentially inappropriate prescribing in residential care using the STOPP/START criteria. Eur Geriatr Med 2016;7:40-46.

31. Nyborg G, Brekke M, Straand J, et al. Potentially inappropriate medication use in nursing homes: An observational study using the NORGEP-NH criteria. BMC Geriatr 2017;17:220.
32. Cox CA, van Jaarsveld HJ, Houterman S, et al. Psychotropic drug prescription and the risk of falls in nursing home residents. J Am Med Dir Assoc 2016;17: 1089-1093.

33. Hartikainen $\mathrm{S}$, Lonnroos $\mathrm{E}$, Louhivuori K. Medication as a risk factor for falls: Critical systematic review. J Gerontol A Biol Sci Med Sci 2007;62:1172-1181.

34. Amos TB, Keith SW, Del Canale $S$, et al. Inappropriate prescribing in a large community-dwelling older population: A focus on prevalence and how it relates to patient and physician characteristics. J Clin Pharm Ther 2015;40:7-13.

35. Al-Jumaili AA, Doucette WR. A systems approach to identify factors influencing adverse drug events in nursing homes. J Am Geriatr Soc; April 24, 2018. https:/ doi.org/10.1111/jgs.15389 [Epub ahead of print]

36. Cullinan S, O'Mahony D, Fleming A, et al. A meta-synthesis of potentially inappropriate prescribing in older patients. Drugs Aging 2014;31:631-638.

37. Spinewine A, Swine C, Dhillon S, et al. Appropriateness of use of medicines in elderly inpatients: Qualitative study. BMJ 2005;331:935.

38. Vlaams indicatorenproject voor woonzorgcentra. Available at: https://www. zorg-en-gezondheid.be/vlaams-indicatorenproject-voor-woonzorgcentravip-wzc. Published 2018. Accessed January 19, 2018.

39. Vlaanderen Expertisecentrum Val- en fractuurpreventie. Praktijkrichtlijn voor woonzorgcentra. Available at:http://www.valpreventie.be/. Published 2018. Accessed January 19, 2018.

40. Hughes CM, Lapane K, Watson MC, et al. Does organisational culture influence prescribing in care homes for older people? A new direction for research. Drugs Aging 2007;24:81-93.

41. San-Jose A, Agusti A, Vidal X, et al. Inappropriate prescribing to the oldest old patients admitted to hospital: Prevalence, most frequently used medicines, and associated factors. BMC Geriatr 2015;15:42.

42. Projovic I, Vukadinovic D, Milovanovic O, et al. Risk factors for potentially inappropriate prescribing to older patients in primary care. Eur J Clin Pharmacol 2016;72:93-107.

43. Gorup EC, Ster MP. Number of medications or number of diseases: What influences underprescribing? Eur J Clin Pharmacol 2017;73:1673-1679.

44. Khodyakov D, Ochoa A, Olivieri-Mui BL, et al. Screening tool of older person's prescriptions/screening tools to alert doctors to right treatment medication criteria modified for U.S. nursing home setting. J Am Geriatr Soc 2017;65:586-591.

45. Lavan AH, Gallagher P, Parsons C, et al. STOPPFrail (Screening Tool of Older Persons Prescriptions in Frail adults with limited life expectancy): Consensus validation. Age Ageing 2017;46:600-607. 